Relations industrielles

Industrial Relations

\title{
Manager Selection, Education and Training, by W.E. Bennett. McGraw-Hill Book Co., New York: 1959, 210 pp.
}

\section{C.-R. Giroux}

Volume 15, numéro 2, avril 1960

URI : https://id.erudit.org/iderudit/1022050ar

DOI : https://doi.org/10.7202/1022050ar

Aller au sommaire du numéro

Éditeur(s)

Département des relations industrielles de l’Université Laval

ISSN

0034-379X (imprimé)

1703-8138 (numérique)

Découvrir la revue

Citer ce compte rendu

Giroux, C.-R. (1960). Compte rendu de [Manager Selection, Education and Training, by W.E. Bennett. McGraw-Hill Book Co., New York: 1959, 210 pp.] Relations industrielles / Industrial Relations, 15(2), 287-289.

https://doi.org/10.7202/1022050ar

Tous droits réservés @ C Département des relations industrielles de l’Université Laval, 1960
Ce document est protégé par la loi sur le droit d'auteur. L'utilisation des services d'Érudit (y compris la reproduction) est assujettie à sa politique d'utilisation que vous pouvez consulter en ligne.

https://apropos.erudit.org/fr/usagers/politique-dutilisation/ 
Manager Selection, Education and Training, by W.E. Bennett. McGraw-Hill Book Co., New York: 1959, 210 pp.

L'entraînement de la direction à tous les niveaux a pris une ampleur incroyable depuis environ dix ans. Toutefois, aucune entreprise ne pèche autant par le manque de logique et de préparation que ces programmes de formation de la gérance. Après deux ans de recherches et d'investigations auprès des grandes compagnies, «l'American Management Association 》 arrive à la conclusion qu'il n'existe pas de procédures ou de méthodes standardisées pour développer le personnel administratif. Une enquête menée par le «National Industrial Conference Board » révèle qu'aucune compagnie n'aborde ce problème de la même façon et n'utilise les mêmes méthodes d'entraînement. Les programmes disparaissent aussi rapidement qu'ils ont débuté. Les entreprises dépensent des sommes énormes pour les élaborer et les conduire. Une activité intense se manifeste à tous les niveaux administratifs, car tous sont d'avis qu'une compagnie ne vaut pas plus que ses gérants et ses administrateurs, et que nombreux sont les gérants qui manquent de connaissances techniques et administratives. Malgré tout, la haute direction est toujours à la recherche d'administratcurs compétents. Pourquoi tant de programmes, élaborés avec un enthousiasme indéniable, ont-ils lamentablement échoué? Pourquoi n'ontils pas obtenu le support des individus qui devaient en profiter? Pourquoi, d'année en année, a-t-on changé «la méthode » d'entraînement sans analyser le contenu du programme?

Personne ne peut répondre à ces questions de façon objective et scientifique. Les chercheurs versés dans les sciences humaines sont encore trop éloignés de ces problèmes concrets, car ils sont trop préoccupés par l'énoncé de théories et de postulats essentiels à la formation d'hypothèses de recherche. Alors, où devions-nous trouver un penseur, un créateur dans ce domaine si ce n'est dans l'industrie même, parmi ceux qui furent aux prises avec les nombreux problèmes d'un programme de développement des cadres administratifs. W.E. Bennett est un des premiers qui a abordé le problème dans toute son ampleur. Son travail révèle une compréhension profonde et complète du problème. Il n'hésite pas à reconsidérer les concepts et les idées dans leur perspective béritable. Actuellement nous assistons dans l'industrie à une diminution de l'importance attribuée aux relations humaines. Pourquoi ? Parce qu'on a voulu s'en servir pour s'assujettir la loyauté des employés, en les manipulant. Bennett considère que le gérant doit envisager les problèmes administratifs causés soit par la production soit par les employés. Ces deux aspects sont aussi importants l'un que l'autre. Dans tout programme d'entraînement de la gérance il faut donc aborder les techniques administratives et les techniques de relations humaines.

Selon l'auteur, la pauvreté des programmes actuels serait imputable à plusieurs causes.

a) La haute direction des compagnies n'a pas su établir et maintenir un climat favorable à l'entraînement.

b) Le gérant et l'administrateur n'ont pas été reconnus comme étant de véritables professionnels.

c) Les personnes entraînées n'ont pas coopéré et ont manifesté une motivation très pauvre.

d) La responsabilité du programme n'a pas été délimitée.

e) La gérance n'était pas convaincue que le coût d'un tel programme devait être considéré comme un coût d'opération.

f) Le directeur du programme changeait trop souvent la méthode d'entrânement, ignorant souvent le matériel à enseigner.

g) La haute gérance n'a pas rendu cet entraînement obligatoire.

h) L'on n'a pas donné à plusieurs la possibilité d'utiliser ce qu'ils avaient appris.

i) Finalement les directeurs des programmes n'ont pas distingué entre l'aspect éducation et l'aspect entraînement.

Au cours du présent ouvrage Bennett s'est déterminé divers objectifs: a) Analyser les facteurs impliqués dans et nécessaires au développement d'un climat propice. 2) Etablir une théorie générale pour la sélection et la formation de la gérance. 3) Suggérer un plan prototype formulé selon les standards et les concepts de cette théorie et suggérant les méthodes et les procédures à suivre pour choisir et former un personnel administratif qui deviendra véritablement pro- 
fessionnel. 4) Considérer les détails de l'administration et de l'exécution du programme. 5) Discuter de l'application des techniques et des programmes recommandés dans le plan prototype. 6) Faire des observations et des commentaires afin de situer le programme dans une perspective réaliste et logique.

Bennett résume ainsi sa conception de la formation des cadres de la gérance:

«Therefore, manager development is, for the most part, on-the-job adult education which must provide formal schooling in the higher theory of the profession as well as a continuing internship of guided and supervised on-the-job training ».

Puisque l'auteur maintient que la gérance est une véritable profession, il faut donc donner aux candidats une éducation théorique et technique et un entralnement pratique; en effet le candidat n'apprend réellement qu'en pratiquant. La plan se déroule en trois étapes: sélection, éducation et entraînement.

LA SELECTION consiste à déterminer dès le début si le candidat possède des qualités de «leader » et surtout, plus important encore, jusqu'à quel degré. L'auteur suggère plusieurs moyens de sélection: un inventaire des aptitudes de la gérance, l'entrevue en profondeur, les tests psychologiques, les promotions temporaires, les notations à la suite d'un entraînement préliminaire, l'analyse du dossier du candidat. Bennett se montre assez sévère à l'égard des tests parce que les praticiens ne s'accordent pas sur la constellation des qualités du «leadership 》 mesurées par eux. N'oublions pas que les tests fournissent des résultats dont l'utilité dépend de l'interprétation donnée et de la théorie du \& leadership » acceptée par l'interprète. Puisque aucune véritable théorie du \&leadership » n'existe actuellement, il ne faut pas en blâmer les tests. D'autre part, Bennet favorise beaucoup la sélection à partir de la notation du personnel, basée sur des traits de personnalité. Or, la recherche a bel et bien prouvé que la sélection et la prédiction du * leadership » à partir de traits n'avait pas beaucoup de validité.

L'EDUCATION est un conditionne- ment intellectuel aux plus importantes théories de la profession et au bagage de connaissances requises par sa pratique. Cette éducation se donne au sein de l'entreprise et à l'extérieur. Selon la matière enseignée, la lecture, la discussion, la conférence, l'étude de cas, etc., sont autant de méthodes qui servent à exposer les principes d'organisation et d'administration, les aspects fonctionnels des affaires, la philosophie de la profession, etc.

L'ENTRAINEMENT représente certainement l'étape la plus importantc. Avant qu'une bonne partie des connaissances acquises ne soit utilisée à l'ouvrage, le caractère de l'individu doit changer de façon drastique. Nous savons tous qu'il est difficile de changer les gens. Ceci requiert souvent de longues séances $d^{\prime}$ analyse. Il faut donc accepter que la facilité de mémoriser des faits et de réussir aux examens écrits n'aura pas beaucoup d'influence sur la performance au travail. L'auteur affirme que la haute direction doit continuellement rendre la gérance responsable de la formation des subalternes à l'ouvrage même. De plus la haute direction doit contrôler cette formation afin de s'assurer que le programme individuel tracé lors de la notation personnelle soit objectif et pratique. La méthode de formation préconisée par l'auteur est la rotation latérale et verticale. Elle permet de former des candidats plus versatiles. Une formation par rotation latérale et vert:cale permet d'obtenir plusieurs notations sur le candidat. Elle augmente les communications entre les divisions et les départements. Elle détruit les mythes de supériorité que s'arrogent certains départements. Elle crée un meilleur climat d'entente.

Le plan suggéré par l'auteur demande une organisation monstre que seules les grandes entreprises peuvent se permettre. En effet, le département de l'entraînement comprendrait lui-même des psychologues, des professeurs et divers administrateurs. Somme toute, le présent ouvrage redonne à l'entraînement de la direction sa véritable importance, parce que Bennett n'hésite pas à bien définir les buts et à bien délimiter les responsabilités impliquées. La gérance et la direction doivent accepter leurs obligations en matière de formation, autrement, l'administration et la direction 
de l'entreprise en souffrira continuellement.

\section{C.-R. Giroux}

La représentativité syndicale, par Jean Neuville. Collection \& Etudes Sociales », nos 31-32. La Pensée catholique. Bruxelles, Office général du livre, Paris 1960, 144 pp.

Le syndicalisme a graduellement réussi à s'imposer devant les employeurs et l'Etat. Dans les pavs à pluralisme syndical, surgit le problème ardu de déterminer quel groupement va représenter les travailleurs au niveau de l'entreprise et de l'industrie comme sur le plan national et international. L'option entre un sustème de représentation majoritaire et relui de la représentation proportionrelle ne fait pas disparaître la difficulté de déterminer le ou les critères de représentativité.

C'est cette question que l'auteur étudie dans la première partie de cet ouvrage. Il v montre que ce que l'on désigne parfois comme critères de représentativité (liberté d'adhésion des membres et indépendanre vis-à-vis des emploveurs. correspondance entre l'aire à renrésenter et les catégoies de membres adhórents, caractère de permanence du sundicat et niveau de cotisations versées par les memhres) ne sont, en réalité. rue des conditions d'antitudes auxrielles devraient répondre les syndicats désirant poser leur candidature à la qualité de «représentatifs ». Le seul critère sérieux de 《représentativité 》 est le nomhre de membres que possède un syndicat.

La seconde partie de l'ouvrage est consacrée à des recherches statistiques sur les effertifs svndicaux belges tentant de suivre l'évolution de la syndicalisation en ce pays.

\section{GÉrARd Dron}

Readings in Unemployment. Prepared for the Special Committee on Unemployment Problems, United States Senate, 86th Congress, 1st Session. U.S. Government Printing Office, Washington 1960, 1716 pp. $\$ 4.50$

Studies in Unemployment. Prepared for the Special Committee on Unemployment Problems, United States Senate, 86th Congress, U.S. Government Printing Office, Washington, 1960. 432 pp. \$1.50.

Nul ne saurait minimiser l'importance du problème du chômage dans notre économie. Il y a, sans doute, un aspect économique et humain. Mais le développement et la survie de notre économie est en jeu. Aussi le Sénat américain a-t-il constitué un comité spécial pour étudier cette question. Les deux ouvrages ci-haut mentionnés ont été préparés pour aider le comité dans son travail.

Le premier est une anthologie de tous les principaux documents et études qui ont été publiés aux Etats-Unis sur le sujet. Il est divisé en trois narties: a) La nolitique publique contre le chômage, histoire, coût et méthodes de mesurer l'emploi et le chômage; b) Causes du chômage et problèmes de groupements particuliers; c) Remèdes.

Le second comprend une série d'études originales préparées par des économistes à la demande du comité sénatorial. On y retrouve les noms des meilleurs experts américains dans cette question.

Ces deux ouvrages valent à eux seuls toute une bibliothèque sur un sujet si vaste et si compliqué. Ils fournissent l'essentiel des données du problème. Sans compter au'ils sont d'un prix très abordable. Nous croyons qu'ils sont indispensables à tout professeur d'économique du travail, tout chef ouvrier et, en somme toute personne qui a à s'occuper de relations du travail.

Gérard Dion 